\title{
La utilidad de los datos georreferenciados con teléfonos móviles para las terapias psicoanalíticas y cognitivo conductuales
}

The usefulness of georeferenced data with mobile phones for geographic analysis and psychoanalytic and cognitive behavioral therapies

\author{
Carlos Ferrás \\ carlos.ferras@usc.es \\ Departamento Xeografía \\ Universidade de Santiago de Compostela (España)
}

\section{Resumen}

Presentamos una reflexión sobre las aportaciones del análisis geográfico y los teléfonos móviles para las terapias psicosociales y sobre cuáles son las preguntas que deben formularse las investigaciones futuras. La producción de datos georreferenciados a través de los teléfonos móviles sobre la vida cotidiana de las personas abre grandes posibilidades para la terapia cognitivo conductual y para el psicoanálisis y la salud mental. Permite elaborar mapas de estados de ánimo que localizan lugares de estrés en la vida diaria de una persona; la cartografía de las emociones, la cartografía cognitiva de los lugares y de nuestros sentimientos.

Palabras clave: Geografía; psicoterapia; teléfonos móviles; terapia cognitivo conductual; psicoanálisis.

\footnotetext{
Abstract

We present a reflection on the contributions of geographic analysis and mobile phones for psychosocial therapies and on the questions that should be asked future research. The production of 
georeferenced data through mobile phones on people's daily lives opens great possibilities for cognitive behavioral therapy and psychoanalysis and mental health. It allows elaborating maps of moods that locate places of stress in the daily life of a person; the mapping of emotions, the cognitive mapping of places and our feelings.

Key words: Geography; psychotherapy; mobile phones; cognitive behavioral therapy; psychoanalysis.

\section{Introducción}

El presente artículo surge del trabajo en equipo que estamos desarrollando en la University of California. Berkeley School of Social Welfare, colaborativamente geógrafos, psicólogos, trabajadores sociales e ingenieros informáticos. ${ }^{1}$ La interacción interdisciplinar nos ha llevado a formular preguntas que necesitan respuesta. Hemos indagado en las bases de datos bibliográficas buscando antecedentes, así como en Internet para identificar proyectos de investigación relacionados.

Hoy en día según datos de la "International Telecomunications Union" (ITU, 2015) a nivel mundial existen 96,8 subscripciones a teléfonos móviles por cada 100 habitantes; con más teléfonos móviles que personas en los países desarrollados (120,6 por cada 100 habitantes) y casi un teléfono por habitante en los países en vías de desarrollo (91,8 por cada 100 habitantes). El teléfono móvil se difunde rápidamente en todo el mundo y se puede convertir en un instrumento de comunicación universal entre las personas. En España se contabilizan 109 subscripciones a teléfonos móviles por cada 100 habitantes y el $80 \%$ de los aparatos son smartphone con acceso a Internet (CNMV, 2015; Fundación Telefónica, 2015).

El teléfono móvil puede capturar datos objetivos para la evaluación psicológica y social (Aguilera, 2015) pero necesita ser concebido como una herramienta de trabajo por parte del profesional y/o terapeuta psicosocial. Desde el punto de vista de la intervención social es preciso considerar que los profesionales del trabajo social no pueden funcionar como unos simples facilitadores de información o gestión de ayudas públicas; los profesionales del trabajo social tienen que orientar sus actividades de intervención con el objetivo de a conseguir la autonomía biopsicosocial de las personas enfermas o en riesgo de exclusión (Hernández, 2013). Además la psicología clínica puede coordinarse y complementarse con el trabajo social creando metodologías de prevención y/o rehabilitación de personas enfermas y/o excluidas. Por su parte, la geografía puede aportar el análisis espacial y la cartografía del contexto, del ambiente social y cultural de los lugares de la vida

\footnotetext{
${ }^{1}$ En el marco de la Beca-Ayuda del programa de estancias en centros de investigación internacionales Salvador de Madariaga del Ministerio de Educación y Ciencia 2016.
} 
cotidiana de las personas, puede elaborar los mapas de la salud mental, de las emociones, del sufrimiento, de la depresión y ansiedad; puede contribuir al diagnóstico biopsicosocial del paciente. Existen grandes posibilidades de aplicar en las terapias psicosociales los resultados de la investigación interdisciplinar entre geógrafos, psicólogos y trabajadores sociales. Partimos de la idea de que la Salud Física y Mental no puede separarse de la Salud Social.

Nuestros objetivos son los siguientes: (1) presentar en estas páginas los resultados de una revisión sistemática de la producción científica más importantes, publicados a nivel internacional e identificados en las bases de datos académico accesibles a través de Internet y a partir de criterios de búsqueda y selección establecidos con antelación; (2) definir cuáles son las orientaciones y preguntas que deben formularse las investigaciones futuras desde el punto de vista del análisis geográfico respecto de la terapia cognitivo conductual y el psicoanálisis.

\section{Método}

Hemos definido palabras clave para buscar los artículos científicos sobre las relaciones entre la geografía y la psicoterapia, utilizando las bases de datos de la Web of Science de Thomson-Reuters que incluye la Social Science Citation Index (SSCI) y, complementado con la búsqueda en el Google Académico. Abarcamos todo el período de tiempo que permite el sistema, no hemos acotado cronológicamente la búsqueda que, según las bases de datos, pueden retraerse en el pasado hasta comienzos del siglo XX.

Las palabras clave utilizadas han sido en inglés "geography", "psychotherapy y "mobile phone" y en español: "geografía", "psicoterapia" y "teléfono móvil"; hemos realizado la búsqueda durante la tercera y cuarta semana del mes de septiembre de 2016. En inglés también hemos introducido "cell phone" en lugar de "mobile phone" por ser una denominación frecuente en el inglés de los Estados Unidos. La búsqueda en la "Web of Science" ha sido por la identificación de alguna combinación de estas palabras o por la presencia de todas ellas en el título, en el tema o en las palabras clave o identificadores de los autores. Hemos buscado artículos científicos, libros y actas de congresos y conferencias. La búsqueda en Google Académico ha sido en los mismos términos pero no ha facilitado mayor información respecto a la obtenida en Web of Science.

Destacar que la búsqueda no ha obtenido resultados con los tres conceptos asociados de geografía, psicoterapia y teléfonos móviles al mismo tiempo. Al contrario hemos encontrado una significativa aunque escasa producción científica con la combinación de los conceptos de geografía y psicoterapia, todo en idioma inglés, y referido a artículos científicos publicados entre el 2005 y el 2016; destacando determinadas revistas médicas en los Estados Unidos como el Journal of Medical Internet Research y la revista del Instituto de Geógrafos Británicos Transations of the Institute of British Geographers, así como la revista Area y la revista Social and Cultural 
Geography. En la búsqueda también destacan dos únicos libros localizados en la Editorial Ashgate en el Reino Unido; uno titulado Emotional Geographies publicado en el 2012 siendo editores Davidson, Smith y Blondi, todo ellos autores que destacan por su producción sobre el mismo tema en las revistas científicas identificadas previamente; y un segundo libro titulado Psychoanalytic Geographies publicado en el 2014 siendo editores Kingsbury y Pile. Hemos descubierto que la primera referencia identificada en Web of Science sobre geografía y psicoterapia data del 2005 y es de Liz Bondi, profesora de geografía social en la Universidad de Edimburgo, también psicóloga y psicoterapeuta. En España no hemos identificado referencias bibliográficas sobre geografía y psicoterapia, destacando como único precedente aislado la referencia de Capel (1999) a una tesis doctoral sobre la localización de la esquizofrenia en Granada.

Por otra parte, hemos completado la búsqueda de la producción científica con una segunda iniciativa. Hemos buscado en Internet a través de Google Académico información asociada a los conceptos de: Mapa de la Depresión, Mapa de la Vulnerabilidad y Mapa de la Salud Mental, encontrando referencias de interés y alcance en los Estados Unidos referida a los Mapas de los Estados de Ánimo, al Mapa de la Vulnerabilidad en España y en las Naciones Unidas referida al Atlas Mundial de la Salud.

\section{Geografía y psicoterapia. Mucha interpretación y poca intervención}

La terapia debemos entenderla como una forma de intervención social que busca la mejora de la salud del paciente. Es preciso diferenciar la terapia psicoanalítica de la terapia cognitiva conductual (TCC) que no son opuestas pero si diferentes en cuanto a sus métodos; el psicoanálisis se basa en el diálogo en sesiones intensas de interacción personal entre paciente y psicoanalista con el fin de analizar el inconsciente y el origen de los problemas mentales para llegar a comprenderlos; por su parte, la terapia cognitiva conductual (TCC) busca la modificación de la conducta negativa para la salud mental en la vida cotidiana del paciente y se base en la formulación y práctica de ejercicios, entrenamiento o aprendizaje de conductas saludables.

Desde el punto de vista de la ciencia geográfica las referencias bibliográficas directas a la psicoterapia son escasas. Bondi (2005), Thien (2005) y Bennett (2009) han reflexionado sobre las conexiones entre la geografía y la psicoterapia; se preguntaron por la relación entre los lugares geográficos y las emociones humanas y como afectan al entorno que les rodea; se formularon interrogantes del tipo: ¿Dónde, en qué lugar, las emociones son sentidas? y ¿Dichas emociones son sentidas en el cuerpo humano y en los lugares geográficos a la vez? ¿Cómo se condicionan e influyen? En Davidson, Smith y Bondi (2012) se define la Geografía de las Emociones y se presentan estudios de casos concretos que buscan la localización de los sentimientos subjetivos de las personas; por ejemplo, la investigación de los lugares donde desean morirse los enfermos de cáncer. Por su parte, MacKian (2007) habla de cartografiar los espacios de la modernidad reflexiva 
y sus paisajes emocionales complejos, para lo cual entrevistó a personas enfermas a largo plazo para conocer la geografía emocional de sus vidas cotidianas.

La geografía y sus relaciones con el psicoanálisis ha sido objeto de reflexiones, análisis e investigaciones diversas; Pile (2009) teorizó sobre las emociones en relación con la geografía y su valor para el psicoanálisis y Curti, Aitken, Bosco y Goerisch (2011) teorizaron el estudio de la afectividad y de los aspectos emocionales de la vida social y personal. Recientemente en Kingsbury y Pile (2014) se desarrolla el concepto de Geografía Psicoanalítica con un enfoque interdisciplinar, donde el concepto de geografía se refiere a la interpretación del espacio social y cultural; es decir, una geografía centrada en el análisis del inconsciente y en la comprensión de las relaciones entre el lugar geográfico y las circunstancias culturales, sociales, económicas y ambientales que originan el desorden, afecciones, o son causa de sufrimientos o malestares psíquicos; en Kingsbury y Pile (2015) comprobamos como 25 investigadores muy diversos, del arte, comunicación, geografía, etc., intentan descubrir cómo el psicoanálisis, el inconsciente y las teorías freudianas, pueden ayudar a entender las cuestiones geográficas y cómo la geografía puede ofrecer nuevos caminos de pensamiento psicoanalítico; sin embargo, no concretan ni definen con precisión sus objetivos y métodos. Por su parte, Kingsbury (2009) reflexiona sobre los métodos psicoanalíticos en la geografía y afirma que no se han desarrollado todavía; observa como el método inductivo y el post-estructuralismo son propios de las terapias del psicoanalista con sus pacientes, el cual a través de métodos como la "libre asociación" de ideas, la "escucha analítica" o la "empatía e identificación" trata de interpretar y comprender el inconsciente del paciente y el origen de sus fobias, traumas o desórdenes mentales.

Existen por tanto teorizaciones y estudios analíticos y descriptivos que evidencian las relaciones entre geografía y psicoterapia cognitiva conductual y psicoanalítica. Sin embargo es necesario avanzar y surgen interrogantes sobre la utilidad de todo ello; ¿cómo estos conceptos y conocimientos pueden facilitar la salud mental? ¿Por qué no se establecen relaciones con la tecnología y con las comunicaciones y los teléfonos móviles? ¿Cómo puede el geógrafo avanzar desde la interpretación y comprensión social y cultural del espacio hacia la intervención psicoterapéutica para prevenir o tratar los problemas de salud mental? ¿Cómo puede la geografía convertirse en una herramienta de intervención psicoterapéutica?

\section{La geolocalización y los mapas de los estados de ánimo}

La cartografía de los estados de ánimo nos permite conocer y comprender la relación entre los lugares geográficos, su contexto social, y sus implicaciones o relaciones mutuas con sentimientos, afectos, comportamientos y experiencias de las personas. Las terapias cognitivas conductuales (TCC) tratan de variar las conductas negativas para la salud física, psíquica y social y, en este contexto ecológico, ambiental, se presentan interesantes posibilidades de intervención 
psicoterapéutica innovadora. Esta cartografía emocional es objeto de investigación desde un enfoque interdisciplinar en el que participan la Inteligencia Artificial y la Psicología, en el cual los teléfonos móviles, sus aplicaciones Apps, acelerómetros y sistemas GPS son las tecnologías claves para la producción y recopilación de datos.

Morris (2010) Ilevó a cabo un estudio de viabilidad de terapias psicosociales a través de teléfonos móviles con un grupo experimental de 8 personas diagnosticadas con síntomas de estrés significativo. Durante un mes dichas personas fueron monitorizadas desde su teléfono produciendo datos sobre su estado de ánimo durante el día, comprobando las variaciones y su referencia geoespacial. Con ello la investigadora pudo estudiar los cambios en el estado de ánimo y su ciclo diurno a nivel espacial, cartografiando los estados de ánimo personales y su análisis ecológicoambiental. Estos mapas permiten diseñar terapias y dar consejo instantáneo en el lugar y momento en el cual la persona sufre un problema emocional.

Por otra parte, desde el Centro de Tecnologías de Intervención Conductual de la Nortwestern University de Chicago, Burns, Begale, Duffecy, Gergle, Karr, Giangrande y Mohr (2011) llevaron a cabo un estudio de viabilidad técnica y fiabilidad funcional de un sistema de atención psicoterapéutica a través del teléfono móvil y la Internet con un pequeño grupo de 8 personas con síntomas de depresión durante 8 semanas. A través de los sensores de los teléfonos móviles, llamadas y mensajes de texto, han sido capaces de detectar automáticamente cuándo y dónde un paciente requiere asistencia. En su estudio, al igual que Morris (2010), han demostrado que los teléfonos móviles pueden facilitar intervenciones al momento en el contexto ambiental y lugar concreto en el cual el paciente necesita ayuda. Fueron capaces de elaborar mapas de estado de ánimo personalizados georreferenciando puntos de colores sobre Google Maps con las coordenadas geográficas facilitadas por los teléfonos móviles, aproximándose a posibles predicciones del estado de ánimo futuro.

Además Mohr, Ho, Duffecy, Reifler, Sokol, Burns, Jin y Siddique (2012) evidencian que los pacientes con depresión prefieren la psicoterapia frente a los medicamentos y que los teléfonos móviles eliminan las barreras de acceso, como puede ser la distancia geográfica, facilitando la participación y el tratamiento psicoterapéutico complementario al tradicional "cara a cara". Es interesante destacar que Dunton, Dzubur e Intille (2016) llevaron a cabo una investigación experimental con un grupo de 39 adolescentes en los Estados Unidos y demostraron, en su caso, que determinados sensores como los acelerómetros en los teléfonos móviles permiten evaluar ambientalmente y en tiempo real la actividad de una persona; permiten conocer la actividad física durante las horas de estudio, de comida, o de ejercicio, e incluso las respuestas emocionales a la actividad física y características contextuales: lugar de la actividad, compañía social y propósito. Asselberg, Ruwaard, Ejdys, Schrader, Sijbrandij y Riper (2016) en los EE. UU. Ilevaron a cabo un 
estudio exploratorio con el teléfono móvil para evaluar el estado de ánimo día a día de un grupo de estudiantes universitarios con síntomas menores de depresión; y pudieron evaluar diariamente y de forma automática los progresos o regresos del estado de ánimo personal.

Actualmente en Internet existen múltiples Apps que ofrecen servicios online de terapia frente a la depresión y ansiedad; Lattie, Schueller, Sargent, Stiles-Shields, Tomasino, Corden, Begale, Karr y Mohr (2016) diseñaron un completo sistema de información llamado "Intellicare" a través de apps en Internet que permiten las terapias online para el tratamiento de la depresión y la ansiedad, y cualquier persona puede acceder libremente descargando dichas aplicaciones en su teléfono móvil, pero solo si cuenta con sistema operativo Android. Dicho sistema ofrece acompañamiento y consejo para prevenir, gestionar y tratar la ansiedad y la depresión, a través de mensajes automáticos diarios al teléfono móvil. Intellicare ha sido diseñado en la Northwestern University de Chicago con subvención del Instituto Nacional de la Salud de los Estados Unidos y hoy en día continúa en desarrollo. Las personas interesadas en recibir terapia a través de Intellicare autorizan el acceso automático a los datos de su teléfono móvil y el monitoreo automático de localización a través de GPS, navegación en Internet, comunicaciones, etc.; y son incentivadas a participar con una compensación directa de 160 dólares. La producción de datos geo-referenciados puede permitir el análisis ambiental y de contexto social de los estados de ánimo, sin embargo, surgen interrogantes sobre la viabilidad y efectividad de este tipo de sistemas inteligentes de terapia para personas vulnerables con dificultades económicas y escasos recursos o capacidades para su uso o acceso.

Las investigaciones desarrolladas demuestran que la cartografía de las emociones personales facilita la psicoterapia, el acceso, la ubiquidad, la superación de las barreras impuestas por la distancia entre pacientes y psicoterapeutas, y ofrece una fuente de información de datos espaciales en mapas de estados de ánimo que permiten analizar las conexiones entre emociones y lugares geográficos, tanto desde el punto de vista ambiental, ecológico, como social.

Sin embargo, desde un punto de vista crítico, debemos observar que los estudios de viabilidad llevados a cabo han sido realizados con pequeños grupos experimentales de estudiantes universitarios y/o jóvenes adolescentes con síntomas leves de depresión o estrés, o con personas incentivadas para participar. Se supone que son grupos de personas voluntarias altamente motivadas con el estudio y muy cualificados en el uso del teléfono móvil; la cuestión que se plantea es hasta qué punto sería posible con personas vulnerables con diferente perfil demográfico, social, económico o cultural. ¿Es viable un servicio de terapia por teléfono móvil en un sistema de salud? Mohr et al. (2012) afirman que la atención primaria es el lugar común para el tratamiento de la depresión y en su estudio con más de 300 pacientes en los Estados Unidos comprobaron que las terapias cognitivo conductuales a través del teléfono móvil obtienen resultados no inferiores a las terapias clásicas cara a cara. Son necesarias nuevas investigaciones en diferentes lugares y con 
diversos grupos sociales para poder evaluar y avanzar en el desarrollo de las terapias móviles personalizadas.

\section{Mobilyze y el análisis forense digintal}

Existen herramientas de recolección de datos y detección electrónica; un software de adquisición, clasificación y análisis de datos desde dispositivos electrónicos como teléfonos móviles con sistemas Windows, Android, Iphone/Ipad y MacOsx. Son herramientas utilizadas por la ciencia forense como práctica para recabar pruebas y datos en procesos legales, y se muestran como posibles herramientas de gran valor para la Geografía Humana y la Psicoterapia. Es un software utilizado por agencias de policía e inteligencia de todo el mundo pero con grandes posibilidades para la investigación social y la salud. Entre las más importantes se encuentran Mobilyze de BlackBag Technologies y Mobile Phone Examiner de Access Data, ambas desarrolladas en el entorno de la Bahía de San Francisco y el Silicon Valley.

Dicho software es capaz de acceder a los datos de cualquier teléfono móvil y elabora informes personalizados. Es capaz de obtener los datos sobre comunicaciones, mensajes, contactos, de voz, texto, etc.; datos sobre las Redes Sociales y los medios de comunicación, fotografías, vídeos, mensajes en Facebook, Twitter, Linkedin, etc; permite obtener los datos georreferenciados de redes wifi conectadas, GeoTags y todo ello geo-localizado con Google Maps; permite conocer los contactos telefónicos, direcciones, mensajes, llamadas ordenadas según un ranking, y permite conocer las aplicaciones utilizadas y la navegación en Internet y su productividad.

Desde el punto de vista de la investigación legal forense, dicho software es aplicado en procesos de investigación judicial o policial. Pero, ¿̇sería posible aplicarlo en terapias psicoterapéuticas? Necesariamente tendría que contar con la autorización y el consentimiento informado del paciente. Sería preciso delimitar claramente el tipo de datos e informes que puede facilitar sin violar la intimidad del propio paciente. Pero, ¿qué tipo de paciente? ¿Con qué tipos de enfermedad mental? Los investigadores sociales y del comportamiento humano debemos tener presente que el teléfono móvil es un lector del estado de ánimo de las personas a través de sistemas Wifi, Bluetooth y GPS que permite datos en tiempo real sobre el contexto ambiental y social del paciente, y el procesamiento de todos estos datos permite el acompañamiento y consejo continuo del terapeuta con su paciente y permite reducir los síntomas de depresión o ansiedad en el momento y lugar preciso.

En la investigación de Saeb, Zhang, Karr, Schueller, Corden, Kording y Mohr (2015) reclutaron 40 personas voluntarias con síntomas de depresión de las cuales 28 aceptaron monitorizar sus teléfonos móviles para facilitar datos continuos de geo-localización a través de GPS y de uso personal del teléfono durante un período de 2 semanas, siendo capaces de establecer 
correlaciones entre estado de ánimo y lugares visitados para facilitar y predecir tratamiento terapéutico; pero se echa en falta un mayor énfasis en el análisis ambiental del lugar identificado.

En este contexto, surge la pregunta de qué puede aportar la Geografía a la Psicoterapia; y, todo indica que los datos geo-referenciados de los pacientes pueden ser producidos, clasificados e interpretados espacialmente y ambientalmente en su contexto por el geógrafo. Pero de nuevo, se platea el reto de la investigación y experimentación al respecto.

\section{Atlas de la vulnerabilidad y mapas de la depresión}

La tecnología móvil y los estudios experimentales en relación con la geografía y la psicoterapia han sido realizados con un enfoque particular de atención individual al paciente. Pero, cंes posible un enfoque comunitario? ¿De qué manera influye el entorno en la salud mental de la comunidad?

En el Atlas Mundial de la Salud de Naciones Unidas se afirma que cuanto mayor es la desigualdad social y la pobreza mayor es el riesgo de enfermedad mental; existe una relación entre vulnerabilidad social y salud mental. La vulnerabilidad en España ha sido cartografiada en un atlas específico, donde a partir de datos oficiales de los Censos de Población y Viviendas de 2001 y 2011 se han localizado los espacios rurales, barrios urbanos y municipios con mayor desigualdad socioeconómica y vulnerabilidad. Se han desarrollado indicadores estadísticos de vulnerabilidad urbanística, índices de desigualdad y análisis contextuales sociales y demográficos que permiten comparaciones entre ciudades, municipios y regiones (Hernández, Matezanz, García, Alguacil, Camacho \& Fernández, 2015; Alguacil, Camacho \& Hernández, 2014).

El Gobierno de los Estados Unidos ha elaborado el Mapa de la Depresión y Estado de Ánimo a partir de dos encuestas telefónicas realizadas en los períodos 1993 a 2001 y 2003 a 2006 a una población estimada de 2,4 millones de adultos. El mapa elaborado muestra el porcentaje de residentes por condado que informaban de un estado de angustia mental frecuente, definido como un malestar emocional durante 14 o más días en el mes anterior a la encuesta, incluyendo el estrés, la depresión y los problemas con la emoción. Moriarty, Zack, Holt, Chapman y Safran (2009) a partir de los datos recabados en dicho Mapa de la Depresión analizan la "geografía de la desesperación", y observan que las comunidades y lugares con mayores niveles de ingresos económicos y educación reportaban menos casos de depresión prolongada o estrés.

En este contexto de producción de datos estadísticos oficiales, como en el caso de España o los Estados Unidos nos surgen más interrogantes: ¿Podemos pensar en terapias psicosociales individuales y comunitarias a medida de barrios urbanos o áreas rurales vulnerables? ¿Podemos localizar geográficamente los lugares de la desesperación? ¿Los sistemas públicos de información estadística pueden ofrecer datos a medida de los programas psicoterapéuticos comunitarios? ¿̇os 
teléfonos móviles pueden ser herramientas de información estadística instantánea? ¿Herramientas de intervención psicoterapéutica geo-localizada?

La observación que hemos llevado a cabo sobre las unidades estadísticas básicas a nivel territorial en los censos de población de los Estados Unidos y España nos permite observar la producción de datos geo-referenciados a diferentes escalas. En los Estados Unidos las unidades estadísticas territoriales menores son los denominados census tract que pueden ser urbanos o rurales y son diseñados como una subdivisión del condado; promedian los 4000 habitantes y oficialmente delimitan áreas geográficas homogéneas respetando los límites de las ciudades, pueblos y asentamientos urbanos o rurales en general; los census tract ofrecen datos sobre características sociales, culturales, económicas y estilo de vida. En el caso de España, la unidad menor estadística a nivel territorial que ofrezca datos similares a los census tract son los municipios; en España los datos a nivel territorial menor procedentes de los censos de población se refieren a los municipios, que a diferencia de los census tract no son unidades homogéneas y su tamaño varía desde unos cientos hasta millones de habitantes y no delimitan ciudades, pueblos o asentamientos urbanos o rurales; existen delimitaciones de áreas censales menores al municipio como pueden ser el distrito electoral, la parroquia o el área censal, pero no ofrecen datos homologables porque no se tratan estadísticamente, no lo permite la Ley de Protección de Datos, o simplemente ofrecen el dato del número de habitantes. Por tanto, existe producción de datos geo-referenciados en los Censos de Población de los Estados Unidos y/o España que podrían ser una fuente de información para el análisis ambiental, contextual, de lo lugares de residencia, de vida diaria, de las personas con problemas de depresión, estrés, o enfermedades mentales en general. Se abren, una vez más, posibilidades para la investigación y la experimentación.

\section{Conclusión}

Las emociones y el comportamiento humano son contextuales y territoriales y están influenciadas por el medioambiente físico y social. Es importante tener presente que la edad, el sexo, la educación, la migración, la movilidad geográfica, la actividad y las condiciones socioeconómicas en un ambiente vulnerable afectan al estado psicológico de las personas y al uso que hacen de los servicios de salud. Las patologías urbanas o rurales como el excesivo ruido, la congestión, el uso del dinero, las relaciones interpersonales, o el aislamiento o soledad generan ansiedades que en determinadas circunstancias darán lugar a los problemas mentales. La producción de datos georreferenciados a través de los teléfonos móviles sobre la vida cotidiana de las personas abre grandes posibilidades para la psicoterapia y la salud mental. Permite elaborar mapas de estados de ánimo personalizados que localizan lugares de mayor o menor estrés en la vida diaria de una persona; permiten la cartografía de las emociones, la cartografía cognitiva de los lugares a los que accedemos físicamente o a través de la Internet, de nuestros sentimientos y experiencias 
psicosociales. Se abren grandes posibilidades para ofrecer tratamientos de psicoterapia personalizados incidiendo en el análisis ecológico-ambiental de los lugares cotidianos de la persona. La difusión de los teléfonos móviles a nivel mundial permite superar barreras de acceso a los servicios de salud cara a cara y generar datos pormenorizados sobre la vida diaria, y la psicoterapia y salud mental pueden llegar a áreas remotas, rurales, y a aquellos espacios socialmente marginados o en riesgo de vulnerabilidad de las ciudades.

La Geografía de las Emociones y Psicoanalítica debe transitar de la teoría a la práctica y facilitar datos espaciales e información georreferenciada útil para el tratamiento terapéutico y la salud mental; puede desarrollar mapas de estados de ánimo con sistemas de geo-localización, puede desarrollar atlas avanzados de las enfermedades mentales, del sufrimiento, de las depresiones y ansiedades; pero también puede recopilar, clasificar, ordenar y producir, datos espaciales sobre los lugares, sobre los espacios vulnerables, y sobre el espacio cotidiano de las personas que sufren los desórdenes mentales. Se abre el debate de cómo y para quién. ¿Es posible la terapia comunitaria dirigida a comunidades vulnerables geo-localizadas en lugares concretos? La tecnología de las comunicaciones móviles, los teléfonos inteligentes, permiten superar las barreras geográficas de la distancia entre el paciente y el terapeuta, permiten la intervención, consejo y acompañamiento, instantáneo en el momento y lugar preciso. Se abre por tanto un gran camino para la investigación interdisciplinar.

La geografía puede aportar a la práctica terapéutica nuevas formas de intervención social para mejorar la salud del paciente. Pero la alianza entre la geografía y la psicoterapia no puede obviar la utilización de los teléfonos móviles y la inteligencia artificial como herramienta de intervención y como dispositivos clave para la producción de datos. El diagnóstico del paciente puede contar con el análisis espacial y la cartografía de los lugares que le puede facilitar la geografía.

Agradecimientos: El presente artículo no sería posible sin la ayuda, debate e intercambio de ideas con Adrián Aguilera (University of California, Berkeley) desde la psicología; con Álvaro Rocha (Universidade de Coimbra) desde la ingeniería informática; con Yolanda García (University of California, Berkeley), desde el trabajo social clínico; y con Magín Borrajo (Petaluma, California) desde la psicoterapia.

Declaración responsable: El autor declara que no existe ningún conflicto de interés en relación a la publicación de este artículo. 


\section{Bibliografía}

Aguilera, A. (2015). Digital technology and mental health intervention: opportunities and challenges. Arbor, 191(771). doi: 10.3989/arbor.2015.771n1012

Asselbergs, J., Ruwaard, J., Ejdys, M., Schrader, N., Sijbrandij, M., \& Riper, H. (2016). Mobile Phone-Based Unobtrusive Ecological Momentary Assessment of Day-to-Day Mood: An Explorative Study. Journal of Medical Internet Research 18(3), e72. doi: 10.2196/jmir.5505

Alguacil, J., Camacho, J., \& Hernández, A. (2014). La vulnerabilidad urbana en España. Identificación y evolución de los barrios vulnerables. Empiria 27, 73-94.

Bennett, K. (2009). Challenging emotions. Area 41(3), 244-251.

Bondi, L. (2005). Making connections and thinking through emotions: between geography and psychotherapy. Transactions of the Institute of British Geographers 30(4), 433-448.

Burns M. N., Begale, M., Duffecy, J., Gergle, D., Karr, C. J., Giangrande, E., \& Mohr, D.C. (2011). Harnessing Context Sensing to Develop a Mobile Intervention for Depression. Journal of Medical Internet Research 13(3), e55.

Capel, H. (1999). [Commentary on the Doctoral dissertation "Estudio geográfico de una población de esquizofrénicos y de sus necesidades en un área de Granada" by Carmen Rosales Varo, presented in Granada in 1998]. Published in Biblio 3W, 144. Retrieved from http://www.ub.edu/geocrit/b3w-144.htm

CNMC (Comisión Nacional de los Mercados y de la Competencia) (2015). Informe económico sectorial de las telecomunicaciones y el audiovisual 2015. Madrid: CNMC. Retrieved from https://www.cnmc.es/expedientes/mtz-2015208

Curti, G. H., Aitken, S., Bosco, F. J., \& Goerisch, D. D. (2011). For not limiting emotional and affectual geographies: a collective critique of Steve Pile's Emotions and affects in recent human geography. Transactions of the Institute of British Geographers, 36, 590-594.

Davidson, J., \& Milligan, C. H. (2004). Embody emotion, sensing space: introducing emotional geographies. Social and Cultural Geography 5(4), 523-532.

Davidson, J., Smith, M., \& Bondi, L. (2012). Emotional Geographies. Aldershot: Ashgate.

Dunton, G.F., Dzubur, E., \& Intille, S. (2016). Feasibility and Performance Test of a Real-Time Sensor-Informed Context-Sensitive Ecological Momentary Assessment to Capture Physical Activity. Journal of Medical Internet Research, 18(6), e106. 
Fundación Telefónica (2015). 16 Informe Sociedad de la Información en España. Madrid: Fundación Telefónica. Retrieved from

http://www.fundaciontelefonica.com/arte_cultura/publicaciones-listado/pagina-itempublicaciones/itempubli/483/

Hernández, A., Matesanz, A., García, C., Alguacil, J., Camacho, J., \& Fernández, C. (2015). Atlas de barrios vulnerables de España: doce ciudades 1991/2001/2006. Madrid: Universidad Politécnica de Madrid, Instituto Juan de Herrera.

Hernández, B. (2013). El Trabajo Social en la intervención psicosocial con personas con trastorno mental severo: una reflexión sobre el papel de las familias. Documentos de Trabajo Social, 52, $314-325$.

ITU (2015). Indicators database. Disponible Mobile-Cellular Telephone Subscription [Data set]. World Telecomm. Indicators 12/2015, 100039398. Retrieved from https://www.itu.int/en/ITUD/Statistics/Pages/publications/wtid.aspx

Kingsbury, P. T. (2009). Psychoanalysis. In R. Kitchin, \& N. Thrift, N. (Eds.), International Encyclopedia of Human Geography, vol. 1, 480-486. Oxford: Elsevier.

Kingsbury, P. Y Pile, S. (2014). Psychoanalytic Geographies. Farnham: Ashgate.

Lattie, E. G., Schueller, S. M., Sargent, E., Stiles-Shields, C., Tomasino, K. N., Corden, M. E., Begale, M., Karr, C. J., \& Mohr, D. C. (2016). Uptake and usage of Intellicare: A publicly available suite of mental health and well-being apps. Internet Interventions 4(2), 152-158. doi: 10.1016/j.invent.2016.06.003

MacKian, S. (2004). Mapping reflexive communities: visualizing the geographies of emotion. Social and Cultural Geography 5(4), 615-631. doi: 10.1080/1464936042000317730

Mohr, D. C., Ho, J., Duffecy, J., Reifler, D., Sokol, L., Burns, M. N., Jin, L. \& Siddique, J. (2012). Effects of Telephone-Administered vs Face to Face Cognitive Behavioral Therapy on Adherence to Therapy and Depression outcomes Primary Care Patients. JAMA, 307(21), 2278-2285. doi: 10.1001/jama.2012.5588

Moriarty, D. G., Zack, M. M., Holt, J. B., Chapman, D. P., \& Safran, M. A. (2009). Geography Patterns of frequent mental distress: US adults 1993-2001 and 2003-2006. American Journal of Preventive Medicine 36(6), 497-505. doi: 10.1016/j.amepre.2009.01.038

Pile, S. (2010). Emotions and effect in recent human geography. Transactions of the Institute of British Geographers, 35, 5-20. doi: 10.1111/j.1475-5661.2009.00368.x 
Saeb, S., Zhang, M., Karr, C. J., Schueller, S. M., Corden, M. E., Kording, K .P., \& Mohr, D. C. (2015). Mobile Phone Sensor Correlates of Depressive Symptom Severity in Daily-Life Behavior: An Exploratory Study. Journal of Medical Internet Research, 17(7), e175. doi: 10.2196/jmir.4273.

Thien, D. (2005). After or beyond feeling? A consideration of affect and emotion in geography. Area, 37(4), 450-454. doi: 10.1111/j.1475-4762.2005.00643a.x 